\title{
Halofuginone-coated urethral catheters prevent periurethral spongiofibrosis in a rat model of urethral injury
}

Krane LS, Gorbachinsky I, Sirintrapun J, Yoo JJ, Atala A, Hodges SJ

Department of Urology, Wake Forest University School of Medicine, Winston-Salem, North Carolina

J Endourol. 2011 Jan 4. [Epub ahead of print]

Background and Purpose: Urethral strictures are from periurethral spongiofibrosis that develops as a result of urethral trauma, disease, or iatrogenic injury. The spongy tissue that surrounds the strictured urethra has an altered ratio of collagen, with increased collagen type I relative to type III. We evaluated the ability of a urethral catheter that was coated with halofuginone (HF), a potent type I collagen inhibitor, to prevent spongiofibrosis formation in a rat model.

Materials and Methods: HF was coated on silicone catheters and release kinetics were measured. Success of impregnation was evaluated with scanning electron microscopy, serial weights, and drug elution data. Urethral strictures were induced in rats using electrocautery. Half the animals had placement of an HF-coated catheter while the others had uncoated silicone controls. Animals were sacrificed at predetermined time points, and urethral tissue was either processed for staining with Masson trichrome and anti-alpha-1 collagen or digested to determine HF concentration. Serum drug levels were also determined in treated animals. Slides were graded by a pathologist who was blinded to treatment to determine collagen deposition.

Results: HF was coated successfully on silicone catheters. Local urethral concentration of HF was tenfold higher than serum concentration in treated rats. Animals with HF-coated catheters had no new type I collagen deposition after urethral injury. Control animals had increased periurethral collagen type I deposition, typical of urethral stricture formation.

Conclusions: HF can be coated successfully on silicone catheters. HF successfully inhibits periurethral type I collagen deposition after urethral injury. This may become an important therapy to prevent urethral stricture formation or recurrence after endoscopic therapy.

\section{Editorial Comment}

The authors present preliminary work in an animal model of urethral injury in which an extract of a Chinese herb is impregnated onto a urethral catheter to prevent build-up of Type 1 collagen. Urethral stricture is known to be associated with accumulation of Type 1 rather than Type 3 collagen in the corpus spongiosum. Halofuginone has been shown to prevent collagen accumulation in other urologic models; however, this is the first study to deliver the compound locally rather than systemically. Indeed, urethral stricture is an attractive field for such a delivery system, in that a urethral catheter provides convenient delivery device. We look forward to future developments in this area.

Dr. Sean P. Elliott

Department of Urology Surgery University of Minnesota

Minneapolis, Minnesota, USA E-mail: selliott@umn.edu 